\title{
Electronic Health Records and Physician Burnout: A Scoping Review
}

\author{
Raghad MUHIYADDIN ${ }^{\mathrm{a}}$, Asma ELFADL ${ }^{\mathrm{a}}$, Ebtehag MOHAMED ${ }^{\mathrm{a}}$, Zubair SHAH ${ }^{\mathrm{a}}$, \\ Tanvir ALAM ${ }^{\mathrm{a}}$, Alaa ABD-ALRAZAQ ${ }^{\mathrm{a}}$ and Mowafa HOUSEH ${ }^{\mathrm{a}, 1}$ \\ ${ }^{a}$ College of Science and Engineering, Hamad Bin Khalifa University, Doha, Qatar
}

\begin{abstract}
This scoping review aims to identify the causes and consequences of physician burnout resulting from using Electronic Health Records (EHRs), as reported by current literature. We identified studies by searching PubMed, Wiley Online Library, and Google Scholar. Study selection and data extraction were conducted by three reviewers independently. Extracted data was then synthesized narratively. Out of 500 references retrieved, 30 studies met all eligibility criteria. We identified six main causes that lead to physician burnout related to the use of EHRs: EHRs' documentation and related tasks, EHRs' poor design, workload, overtime work, inbox alerts, and alert fatigue. We also identified the following consequences of physician burnout: low-quality care, behavioral issues, mental health complications, substance abuse, career dissatisfaction, costly turnover, and a decline in patient safety and satisfaction.
\end{abstract}

Keywords. Physicians, Electronic Health Records, Electronic Medical Records, burnout.

\section{Introduction}

The Electronic Health Record (EHR) is an advanced version of paper-based health records wherein a patient's information is collected and stored digitally [1]. EHRs are an essential component in the e-health domain, and the demand for interaction with electronic systems is increasing. Although using EHRs is associated with many advantages, they have now been identified to be one of the main reasons for physician burnout [2]. Burnout, which often occurs when physicians are faced with long-term stress, may cause depersonalization, emotional exhaustion, and the absence of a sense of selfachievement [3]. The impact of EHRs on physician burnout is an important topic, as it affects many physicians. This phenomenon and subsequently may endanger both their lives as well as the lives of the patients they are treating. Many studies examined how EHRs lead to physician burnout, but only a few reviews thoroughly covered all aspects of this topic. Specifically, the existing reviews mainly focused on physician burnout in general or gave an overview of the negative aspects of EHRs. Thus, the objective of this scoping review is to identify the causes and consequences of physician burnout as a result of using EHRs as reported by the current literature.

${ }^{1}$ Corresponding Author, Dr. Mowafa Househ, College of Science and Engineering, Hamad Bin Khalifa University, Doha, Qatar; Email: mhouseh@hbku.edu.qa. 


\section{Methods}

This scoping review was conducted in line with the Joanna Briggs Institute guidelines. We searched three different databases: PubMed, Wiley Online Library, and Google Scholar. The study selection process consisted of three steps: removing the duplicates in the 500 retrieved studies, screening titles and abstracts of the studies, and reading the full text of the remaining studies (Appendix 1). We then extracted data into an Excel sheet, which was synthesized using a narrative approach. Then, all included papers were summarized into simplified text and tables.

\section{Results}

A total of 30 studies were included out of the 500 retrieved studies. The included studies reported six main causes of how EHRs lead to physician burnout. These causes were EHRs' documentation and related tasks, EHRs' poor design, workload, work overtime, inbox alerts, and alert fatigue. Eight studies addressed burnout factors stemming from indirect patient care tasks such as documentation, note-taking, and electronic communication in EHRs [4-11]. Nine studies found that EHRs' difficult use and poor design were associated with physician stress and burnout [6,12-19], where EHRs with a high number of functions are likely to have more stress on them than those with fewer functions [19]. Fourteen studies discussed how the increase in workload in EHRs increased burnout $[4,10,15,18-28]$. Four studies examined how physicians felt burned out due to the workload that results from using EHRs, which led to working overtime during evenings and weekends at home $[8,13,25,29]$. Six studies explored how inbox alerts from EHRs, including medication refills and test results, caused alert fatigue and eventual physician burnout $[4,5,23,24,29,30]$.

Nine studies discussed the consequences of EHR burnout; namely, that physicians could not provide quality care, which ultimately results in global degradation of the quality of care [4,5,13,19,20,25,31-33]. Burnout affects physicians' behavior [31], including that some burned-out physicians may behave unprofessionally towards their colleagues [11,28]. Additionally, burnout affects the health of the physician [31].Physicians experiencing burnout are more likely to have mental problems, including depression, which can lead to suicide $[25,28,33]$. They are also more prone to substance abuse disorders [11,32]. A physician's burnout also affects patients' safety and satisfaction [31]. Patients treated by burned-out physicians were dissatisfied with their care, as they experience medical errors and unneeded assessments $[11,28,30,31,33]$. In general, burned-out physicians are not satisfied with their careers [31]. Lastly, burnout leads to costly turnover if the physician decides to leave as it is costly to replace them $[11,30]$.

\section{Discussion}

This scoping review summarizes the studies that focus on physician burnout related to EHRs, as well as the causes and consequences of burnout. Two reviews discussed how the United States' physicians now spend more time on their computers than they do interacting with their patients $[16,32,33]$. Our findings align with previous reviews, which discuss that physicians are now communicating through portals about their 
patients' concerns. They describe this as "doctoring while typing" clinical workflow, which leads to an overload of information and extra time spent on documentation; all worsening the physician's satisfaction [33-36]. Another similar review also found that physicians suffering from alert fatigue and must ignore reminders that pop-up on their screen to combat this [37].

\section{Conclusion}

The paper's cited reviews have found that EHRs are the leading cause of physician burnout. Many causes lead to physicians who use EHRs feeling burnt out, including as the necessary documentation and related tasks, poor design of EHRs, extra workload and working overtime, constant inbox alerts, and alert fatigue. There were also consequences to burnout such as low-quality care, physician dissatisfaction, turnover, mental health issues, increased substance abuse, behavioral problems, and patient dissatisfaction. We recommend researchers conduct this research to increase efforts in finding ways to relieve physician burnout. We advise them to identify the causes and consequences of burnout resulting from using EHRs by other health providers such as nurses, pharmacists, and technicians. Appendix files are available at Github: https://github.com/rmuhiyaddin/Appendix...git.

\section{References}

[1] HealthIT.gov. What Is an Electronic Health Record (EHR)?. 10 Sept. 2019. Available from: https://www.healthit.gov/faq/what-electronic-health-record-ehr.

[2] Beresford, Larry. Research Shows Link Between EHR and Physician Burnout. The Hospitalist.

[3] AHRQ. Physician Burnout. July 2017. Available from: https://www.ahrq.gov/prevention/clinician/ahrqworks/burnout/index.html.

[4] Tran, Brian, et al. Burnout and EHR Use among Academic Primary Care Physicians with Varied Clinical Workloads. AMIA Joint Summits on Translational Science Proceedings. AMIA Joint Summits on Translational Science, 2019;2019:136-144.

[5] Wang, Jason K., et al. Characterizing Electronic Health Record Usage Patterns of Inpatient Medicine Residents Using Event Log Data. PLOS ONE. 6 Feb. 2019;14(2):e0205379.

[6] Hauer A. et al. Physician Burnout in Wisconsin: An Alarming Trend Affecting Physician Wellness. WMJ : Official Publication of the State Medical Society of Wisconsin, 2018;117(5):194-200.

[7] Tutty, Michael A, et al. The Complex Case of EHRs: Examining the Factors Impacting the EHR User Experience. Journal of the American Medical Informatics Association. 2 Apr. 2019;26(7):673-677.

[8] Robertson SL, Robinson MD, Reid A. Electronic health record effects on work-life balance and burnout within the I3 population collaborative. Journal of graduate medical education. 2017 Aug;9(4):479-84.

[9] Chen L. et al. Racing Against the Clock: Internal Medicine Residents' Time Spent On Electronic Health Records. Journal of Graduate Medical Education. Feb. 2016;8(1):39-44, 10.4300/jgme-d-15-00240.1.

[10] Reisman M. EHRs: The Challenge of Making Electronic Data Usable and Interoperable. P \& T : A PeerReviewed Journal for Formulary Management. 2017;42(9):572-575.

[11] Gardner RL, et al. Physician Stress and Burnout: The Impact of Health Information Technology. Journal of the American Medical Informatics Association. 5 Dec. 2018;26(2):106-114, 10.1093/jamia/ocy145.

[12] Friedberg MW. et al. Factors Affecting Physician Professional Satisfaction and Their Implications for Patient Care, Health Systems, and Health Policy. Rand Health Quarterly. 2014;3(4):1.

[13] Kroth PJ, et al. The Electronic Elephant in the Room: Physicians and the Electronic Health Record. JAMIA Open. 11 June 2018;1(1):49-56.

[14] Fred HL, Scheid MS. Physician burnout: causes, consequences, and (?) cures. Texas Heart Institute Journal. 2018 Aug;45(4):198.

[15] Domaney NM, et al. Exploring the Association Between Electronic Health Record Use and Burnout Among Psychiatry Residents and Faculty: A Pilot Survey Study. Academic Psychiatry. 21 May 2018;42(5):648-652. 
[16] Alexander AG, Ballou KA. Work-life balance, burnout, and the electronic health record. The American journal of medicine. 2018 Aug 1;131(8):857-8.

[17] Kroth PJ, et al. Association of Electronic Health Record Design and Use Factors With Clinician Stress and Burnout. JAMA Network Open. 16 Aug. 2019;2(8):e199609.

[18] Heponiemi T, et al. Predictors of Physicians' Stress Related to Information Systems: A Nine-Year Follow-up Survey Study. BMC Health Services Research. 13 Apr. 2018;18(1).

[19] Babbott S, et al. Electronic Medical Records and Physician Stress in Primary Care: Results from the MEMO Study. Journal of the American Medical Informatics Association. Feb. 2014;12(e1):e100-e106. 10.1136/amiajnl-2013-001875.

[20] Arndt BG, et al. Tethered to the EHR: Primary Care Physician Workload Assessment Using EHR Event Log Data and Time-Motion Observations. The Annals of Family Medicine. Sept. 2017;15(5):419-426. $10.1370 / \mathrm{afm} .2121$.

[21] Robinson KE, Kersey JA. Novel electronic health record (EHR) education intervention in large healthcare organization improves quality, efficiency, time, and impact on burnout. Medicine. 2018 Sep;97(38).

[22] Roberts DL, Shanafelt TD, Dyrbye LN, West CP. A national comparison of burnout and work - life balance among internal medicine hospitalists and outpatient general internists. Journal of hospital medicine. 2014 Mar;9(3):176-81.

[23] Gilleland M, Komis K, Chawla S, Fernandez S, Fishman M, Adams M. Resident duty hours in the outpatient electronic health record era: inaccuracies and implications. Journal of graduate medical education. 2014 Mar;6(1):151-4.

[24] Khairat S, et al. Focus Section on Health IT Usability: Perceived Burden of EHRs on Physicians at Different Stages of Their Career. Applied Clinical Informatics. Apr. 2018;9(02):336-347.

[25] Khairat S, et al. Physicians' Gender and Their Use of Electronic Health Records: Findings from a MixedMethods Usability Study. Journal of the American Medical Informatics Association 28 Aug. 2019;26(12). 10.1093/jamia/ocz126.

[26] Rassolian M, Peterson LE, Fang B, Knight HC, Peabody MR, Baxley EG, Mainous AG. Workplace factors associated with burnout of family physicians. JAMA internal medicine. 2017 Jul 1;177(7):10368.

[27] Privitera MR, et al. Physicians' Electronic Health Records Use at Home, Job Satisfaction, Job Stress and Burnout. Journal of Hospital Administration 20 June 2018;7(4): 52.

[28] Ripp JA, et al. Well-Being in Graduate Medical Education. Academic Medicine. July 2017:92(7):914917.

[29] Russo E, et al. Electronic Health Record Alert-Related Workload as a Predictor of Burnout in Primary Care Providers. Applied Clinical Informatics. 2017;08(03):686-697.

[30] Meigs SL, Solomon M. Electronic health record use a bitter pill for many physicians. Perspectives in health information management. 2016;13(Winter).

[31] Marckini DN, et al. Electronic Health Record Associated Stress: A Survey Study of Adult Congenital Heart Disease Specialists. Congenital Heart Disease. Mar. 2019;14(3):356-361.

[32] Stehman C, et al. Burnout, Drop Out, Suicide: Physician Loss in Emergency Medicine, Part I. Western Journal of Emergency Medicine. 23 Apr. 2019;20(3):485-494.

[33] Kuhn CM, Flanagan EM. Self-Care as a Professional Imperative: Physician Burnout, Depression, and Suicide. Canadian Journal of Anesthesia/Journal Canadien d'anesthésie. 1 Dec. 2016;64(2):158-168. 10.1007/s12630-016-0781-0.

[34] Ratanawongsa N, et al. Reducing Misses and Near Misses Related to Multitasking on the Electronic Health Record: Observational Study and Qualitative Analysis. JMIR Human Factors. 6 Feb. 2018;5(1):e4.

[35] Bodenheimer T, Sinsky C. From Triple to Quadruple Aim: Care of the Patient R7quires Care of the Provider. The Annals of Family Medicine. 1 Nov. 2014;12(6):573-576. 10.1370/afm.1713.

[36] Houkes I, Winants Y, Twellaar M, Verdonk P. Development of burnout over time and the causal order of the three dimensions of burnout among male and female GPs. A three-wave panel study. BMC Public health. 2011 Dec;11(1):1-3.

[37] Backman R, et al. Clinical Reminder Alert Fatigue in Healthcare: A Systematic Literature Review Protocol Using Qualitative Evidence. Systematic Reviews. Dec. 2017;6(1). 10.1186/s13643-017-0627z. Accessed 12 Dec. 2019. 